\title{
Specification of New Test Waveforms for Static Electricity
} Meters

This paper was downloaded from TechRxiv (https://www.techrxiv.org).

\section{LICENSE}

CC BY 4.0

SUBMISSION DATE / POSTED DATE

07-01-2021 / 08-01-2021

CITATION

Ritzmann, Deborah; Wright, Paul (2021): Specification of New Test Waveforms for Static Electricity Meters. TechRxiv. Preprint. https://doi.org/10.36227/techrxiv.13536899.v1

DOI

10.36227/techrxiv.13536899.v1 


\title{
Specification of New Test Waveforms for Static Electricity Meters
}

\author{
Deborah Ritzmann and Paul Wright \\ National Physical Laboratory, UK \\ deborah.rizmann@npl.co.uk
}

\begin{abstract}
Recent observations of significant errors in static electricity meter readings have created a need for specification of new standard immunity test waveforms. This paper provides an overview of ongoing signal analysis work towards parsimonious specification of suitable test waveforms including candidate methods for signal representation, feature extraction and feature selection.
\end{abstract}

Index Terms - Electromagnetic compatibility, energy measurement, immunity testing, measurement standards, meter reading, watthour meters.

\section{INTRODUCTION}

Across the world, electromechanical electricity meters are being replaced with static electricity meters to exploit the benefits of communication capabilities. In recent years several cases of errors in type-approved meter readings have been reported and are suspected to be caused by conduced interference [1]. The pre-normative research project MeterEMI is developing a test framework to determine the extent of this problem and provide input to relevant testing standards [2], [3].

An important part of this framework is the specification of test waveform features to ensure immunity against meter errors. As a first step towards this specification, the error sensitivity of static electricity meters has been analyzed experimentally with different load currents [4]. The slope, rise time, rising edge and charge have been considered as relevant error-inducing time domain features. The next step is a sparse mathematical representation for the pertinent waveform features. This paper presents an overview of available signal models and decompositions that are under consideration, along with a discussion of relevant feature extraction and selection methods for identifying signal features with the highest sensitivity to cause meter errors.

\section{METHOdS FOR SignAL REPRESENTATION AND FEATURE EXTRACTION}

A mathematical representation is required in order to specify waveforms for immunity testing of electricity meters. The representation should be sparse, with a small number of required parameters that are linked to observed causes of electricity meter errors.

Parametric closed-form models can give compact signal representation but require a priori assumptions about the waveforms. Errors in electricity meter readings have been observed for a range of linear and non-linear loads with different non-sinusoidal current waveforms [4]. Therefore, non-parametric signal processing tools provide a more general basis for analysis. The number of features resulting from a nonparametric signal decomposition can be greater than the size of the length of the input time series, which creates the challenge of identifying salient features with respect to the phenomenon of interest. Feature extraction is the process of combining features, with the aims of creating a new feature which is a stronger predictor and to reduce the overall number of features to study [5]. The candidate methods under consideration are discussed in the rest of this section.

\section{A. Discrete Fourier Transform}

The Discrete Fourier Transform (DFT) is a candidate representation because it is a common, efficient and wellunderstood tool used by power system engineers. The sinusoidal basis functions compactly capture the main frequency characteristics of steady-state voltage and current waveforms. However, non-sinusoidal load waveforms with sharp transitions can result in broad spectra with diverse frequency content. To reduce the number of features a representative metric such as total harmonic distortion can be calculated.

\section{B. Wavelet Transforms}

The wavelet transform decomposes a signal using scaled and shifted versions of a short-duration basis wave function (mother wavelet). Instead of a sinusoidal basis, a pulse-like wave function can be chosen to match error-inducing waveforms. One advantage over the DFT is that the frequency resolution of the wavelet transform is not fixed. Low frequency slow phenomena can be characterized by coefficients with high frequency resolution whereas short-duration high frequency signal parts can be analyzed with high time resolution.

The Continuous Wavelet Transform gives a shift-invariant, redundant representation due to overlapping scaling and shifting of the mother wavelet. The resulting coefficients form a finely meshed grid of scales with small time steps, which can be useful for detailed analysis of transient signals. To reduce the resulting feature space of coefficients, calculation of a dominant frequency signature has been proposed based on the maximum coefficient value at each instant [6].

The advantage of the Discrete Wavelet Transform is that it is a sparser, energy-preserving decomposition without overlap. The waveform is decomposed by iteratively dividing the bandwidth using low-pass and high-pass filters. At each filtering step the high-pass band gives a new set of detail coefficients describing fast-changing signal features, while the low frequency band is downsampled, further decomposed and 
ultimately results in approximation coefficients describing the smooth, slow signal features. The wavelet coefficients form a feature space from which new features can be extracted for each level of decomposition such as energy content, entropy, maximum value, kurtosis or crest factor [7].

\section{Quadratic Time-frequency Distributions}

The Fourier and Wavelet Transforms depend linearly on the input waveform. Another class of distributions are those that depend quadratically on the input signal [8]. The properties of the quadratic time-frequency distribution are determined by the constraints that are placed on the transform kernel function. Commonly, the kernel function is chosen to satisfy marginal conditions, i.e. the integral of the power spectrum equals total signal energy, giving an energetic interpretation of the timefrequency spectrum. The kernel function can also be chosen as a characteristic signal feature [9].

\section{METHODS FOR FEATURE SELECTION}

The signal decomposition techniques discussed in the previous section generate a range of features. Feature selection methods will be used to create a subset of features that are most relevant to errors in static electricity meters by discarding redundant and non-error inducing features [5].

A feature selection algorithm searches for candidate subsets from the available feature space and evaluates the relevance of each subset to static meter errors using an objective function. The search for candidate subsets can be exhaustive, sequential or random [10]. An exhaustive search tests every possible subset and finds the most relevant features at exponential cost; a sequential search is more efficient by incrementally extending or reducing the feature subset according to the result of the objective function, while a random search for feature subsets avoids getting stuck on local minima during optimization of the objective function.

A predictor model could be trained to relate signal feature values to static meter errors, and the objective function could be based on prediction accuracy to evaluate feature relevance (wrapper objective function). On the other hand, filter objective functions do not require a predictor model as relevance is evaluated based only on feature values. An example is Fisher's discriminant which assigns a higher score to features that "maximize distances between the means of the different classes while minimizing the variances within each class" [9]. The selection of relevant features for static meter test signal specification will be initially based on a sequential search algorithm in combination with a filter objective function.

\section{CONCLUSION}

This paper is a summary of ongoing signal analysis work to specify new standard test waveforms for static electricity meters to ensure immunity against conducted interference.
A range of candidate methods for signal representation, feature extraction and feature selection have been discussed. Implementation, evaluation and optimization of these methods on measured waveform data is in progress. New test waveforms will be specified based on the most optimal representation and features; the ability of the waveforms to imitate loads that cause meter errors will be validated experimentally. Results will be presented at the conference.

\section{ACKNOWLEDGEMENT}

This work is carried out within the project 17NRM02 MeterEMI, which has received funding from the EMPIR program co-financed by the Participating States and from the European Union's Horizon 2020 research and innovation program.

\section{REFERENCES}

[1] B. ten Have, T. Hartman, N. Moonen, and F. Leferink, "Misreadings of static energy meters due to conducted EMI caused by fast changing current," in Joint International Symposium on Electromagnetic Compatibility, Sapporo and Asia-Pacific International Symposium on Electromagnetic Compatibility (EMC Sapporo/APEMC), 2019, pp. 445-448.

[2] P. S. Wright et al., "Evaluation of EMI effects on static electricity meters," in Conference on Precision Electromagnetic Measurements (CPEM), 2018, pp. 1-2.

[3] Electromagnetic compatibility (EMC) - Part 4-19: Testing and measurement techniques - Test for immunity to conducted, differential mode disturbances and signalling in the frequency range. IEC 61000-4-19:2014 Ed. 1.0, 2014.

[4] Z. Marais, H. E. Van Den Brom, G. Rietveld, R. Van Leeuwen, D. Hoogenboom, and J. Rens, "Sensitivity of static energy meter reading errors to changes in non-sinusoidal load conditions," in EMC Europe - International Symposium on Electromagnetic Compatibility, 2019, pp. 202-207.

[5] J. Tang, S. Alelyani, and H. Liu, "Feature selection for classification: a review," in Data Classification: Algorithms and Applications, Chapman and Hall/CRC, 2014, pp. 511-536.

[6] S. Chatterjee, S. Chakravorti, C. K. Roy, and D. Dey, "Wavelet network-based classification of transients using dominant frequency signature," Electr. Power Syst. Res., vol. 78, no. 1, pp. 21-29, Jan. 2008.

[7] H. Erişti, A. Uçar, and Y. Demir, "Wavelet-based feature extraction and selection for classification of power system disturbances using support vector machines," Electr. Power Syst. Res., vol. 80, no. 7, pp. 743-752, Jul. 2010.

[8] L. Cohen, "Time-frequency distributions-a review," Proc. IEEE, vol. 77, no. 7, pp. 941-981, Jul. 1989.

[9] M. Wang and A. V. Mamishev, "Classification of power quality events using optimal time-frequency representations - Part 1: Theory," IEEE Trans. Power Deliv., vol. 19, no. 3, pp. 14881495, Jul. 2004.

[10] L. C. Molina, L. Belanche, and A. Nebot, "Feature selection algorithms: a survey and experimental evaluation," in IEEE International Conference on Data Mining, 2002. Proceedings., 2002, pp. 306-313. 